\title{
APPROXIMATE SOLUTIONS AND EXISTENCE OF SOLUTION FOR A CAPUTO NONLOCAL FRACTIONAL VOLTERRA FREDHOLM INTEGRO-DIFFERENTIAL EQUATION
}

\author{
Basim N. Abood \\ Department of Mathematics \\ College of Education of Pure Science \\ University of Wasit, IRAQ
}

\begin{abstract}
We use a recent approach to establish the existence and uniqueness results of Caputo fractional Volterra Fredholm integro-differential equation. We derive some sufficient conditions for the existence of solutions of fractional integrodifferential equations with nonlocal conditions. the modified Adomian decomposition method is applied to obtain the approximate solution of proposed problem. Moreover, the Krasnoselskii's and Banach's fixed point theorems are employed to analyze our results. An example is given to justify the adduced results.
\end{abstract}

AMS Subject Classification: 34K37, 26A33, 34A12, 47H10

Key Words: fractional integrodifferential equations; Caputo fractional derivative; existence theorem; modified Adomian decomposition method; fixed point theorem

\section{Introduction}

In this work, we study the existence and uniqueness of solution by using some fixed point theorems of Krasnoselskii and Banach, then we apply the modified Adomian decomposition method (MADM) for the following Caputo fractional Volterra Fredholm Integro-Differential Equation (Caputo fractional VFIDE)

Received: June 19, 2020

(C) 2020 Academic Publications 


$$
\left\{\begin{array}{c}
{ }^{C} D_{0^{+}}^{\rho} x(t)=g(t)+\chi_{1} x(t)+\chi_{2} x(t), t \in I=[0,1], \\
x(0)=x_{0}+h(x),
\end{array}\right.
$$

where $0<\rho<1,{ }^{C} D_{0^{+}}^{\rho}$ is fractional derivative of order $\rho$ in the Caputo sense, $g: I \rightarrow \mathbb{R}, h: C(I, \mathbb{R}) \rightarrow \mathbb{R}, K_{1}, K_{2}: I \times I \rightarrow \mathbb{R}$ are continuous functions and $F_{1}, F_{2}: \mathbb{R} \rightarrow \mathbb{R}, i=1,2$ are Lipschitz continuous functions. For short, we set

$$
\chi_{1} x(t):=\int_{0}^{t} K_{1}(t, \xi) F_{1}(x(\xi)) d \xi
$$

and

$$
\chi_{2} x(t):=\int_{0}^{1} K_{2}(t, \xi) F_{2}(x(\xi)) d \xi .
$$

In recent years, the fractional integrodifferential equation emerges in a lot of phenomena of different fields of science and engineering, [9, 22, 21, 24].

Some results on the existence of solutions of fractional integrodifferential equations have been studied by many authors by employing the fixed point techniques. For recent papers, see $[1,2,3,4,5,6,31,11,14,16,20,10,29,30,25,32]$. Furthermore, much researches on the approximate solution of this kind of equations have occurred through the method of Adomian decomposition introduced by George Adomian [7] and other numerical methods for more details see $[8,19,15,18,34]$. The method of Adomian decomposition has the feature of style and easiness of use. The solution is provided as a series in which every expression can be easily calculated by means of Adomian polynomials appropriated to nonlinear terms see [7, 12, 13, 17, 26, 27].

Wazwaz in [33] presented the method of modified Adomian decomposition (MADM) that contains decomposing the $1^{\text {st }}$ term of the series into $2^{\text {nd }}$ terms, one remains in the $1^{\text {st }}$ term while the other is assigned to define the $2^{\text {nd }}$ term of series. The main aim of this method is to reduce the number of operations used and quicken the convergence towards the exact solution of the proposed problem. For instance on the application of the MADM, we refer to [23].

In this article, we use a recent approach to establish the existence and uniqueness results of Caputo fractional VFIDE (1). We derive some sufficient conditions for the existence of solutions of fractional integrodifferential equations with nonlocal conditions. the MADM is applied to obtain the approximate solution of Moreover, the Krasnoselskii's and Banach's fixed point theorems are employed to analyze our results.

The paper is organized as follows. In Section 2, we give some basic results related the hypothesis and several lemmas needed throughout this work. In Section 3, we prove the existence and uniqueness of solutions to the porposed 
problem by means of fixed point theorems of Krasnoselskii and Banach. In section 4, we discuss the modified Adomian decomposition method and establish the convergence of the series built by the MADM to the exact solution of the Caputo fractional VFIDE. Finally, we give an example to illustrate our results.

\section{Preliminaries}

In this section, we need the following basic definitions and Lemmas used throughout this paper. For more details, see [24].

Definition 1. Let $\rho>0$, and $\omega \in L^{1}([0, T], \mathbb{R})$. The Riemann-Liouville fractional integral of order $\rho$ is defined by

$$
I_{0^{+}}^{\rho} \omega(t)=\left\{\begin{array}{lc}
\frac{1}{\Gamma(\rho)} \int_{0}^{t}(t-\xi)^{\rho-1} \omega(\xi) d \xi, & \rho>0 \\
\omega(t), & \rho=0
\end{array}\right.
$$

where $\Gamma$ is the Euler's Gamma function satisfies

$$
\Gamma(\rho)=\int_{0}^{\infty} t^{\rho-1} e^{-t} d t, \text { and } \frac{\Gamma(\rho) \Gamma(\beta)}{\Gamma(\rho+\beta)}=\int_{0}^{1}(1-t)^{\rho-1} t^{\beta-1} d t .
$$

Moreover, The operator $I_{0^{+}}^{\rho}$ is bounded on $C([0, T], \mathbb{R})$, i.e., for a positive constant $\kappa$

$$
\left\|I_{0^{+}}^{\rho} \omega\right\|_{\infty} \leq \kappa\|\omega\|_{\infty}, \text { for all } \omega \in C([0, T], \mathbb{R}) .
$$

Definition 2. Let $\rho>0, \omega \in A C^{n}([0, T], \mathbb{R})$. The Caputo fractional derivative of order $\rho$ is defined by

$$
{ }^{C} D_{0^{+}}^{\rho} \omega(t)=D_{0^{+}}^{\rho}\left[\omega(t)-\sum_{k=0}^{n-1} \frac{\omega^{(k)}(0)}{k !} t^{k}\right] \quad t \in[0, T],
$$

where $n=[\rho]+1,[\rho]$ is the integer part of $\rho$ and $D_{0^{+}}^{\rho}$ is the fractional derivative of order $\rho$ in the Riemann-Liouville sense defined by

$$
\begin{aligned}
D_{0^{+}}^{\rho} \omega(t) & =\left(\frac{d}{d t}\right)^{n} I_{0^{+}}^{n-\rho} \omega(t) \\
& =\left(\frac{d}{d t}\right)^{n} \frac{1}{\Gamma(n-\rho)} \int_{0}^{t}(t-\xi)^{n-\rho-1} \omega(\xi) d \xi
\end{aligned}
$$


Lemma 1. If $\rho, \beta>0$, then

$$
I_{0^{+}}^{\rho} t^{\beta-1}=\frac{\Gamma(\beta)}{\Gamma(\rho+\beta)} t^{\rho+\beta-1} .
$$

Lemma 2. Let $\omega \in A C^{n}(I, \mathbb{R})$, then the Caputo fractional derivative of order $\rho>0$

$$
I_{0^{+}}^{\rho}{ }^{C} D_{0^{+}}^{\rho} \omega(t)=\omega(t)-\sum_{k=0}^{n-1} \frac{\omega^{(k)}(0)}{k !} t^{k},
$$

where $n=[\rho]+1$. In a special case, if $0<\rho<1$, then $I_{0^{+}}^{\rho}{ }^{C} D_{0^{+}}^{\rho} \omega(t)=\omega(t)-$ $\omega(0)$. Furthermore, if $\omega$ is a continuous on $I$, we have ${ }^{C} D_{0^{+}}^{\rho} I_{0^{+}}^{\rho} f(t)=f(t)$.

Theorem 3. ([28]) (Banach fixed point theorem) Let $(U, d)$ be a Banach space with $T: U \rightarrow U$ is a contraction mapping. Then mapping $T$ has a fixed point in $U$.

Theorem 4. ([28]) (Krasnoselskii fixed point theorem) Let $U$ be a Banach space, let $S$ be a nonempty bounded closed convex subset of $U$ and let $T_{1}, T_{2}$ be mapping from $S$ into $U$ such that $T_{1} x+T_{2} v \in S$ for any $x, v \in S$. If $T_{1}$ is contraction and $T_{2}$ is completely continuous, then the equation $T_{1} z+T_{2} z=z$ has a solution on $S$.

\section{Existence result via Krasnoselkii's fixed point theorem}

In this part, we study existence of solution of Caputo fractional VFIDE (1) by using Krasnoselkii's fixed point theorem.

First we make the following assumptions.

$\left(\mathbf{H}_{1}\right)$ Let $F_{1}(x(t)), F_{2}(x(t))$ can be considered as continuous nonlinearity terms and there exist constants $L_{F_{1}}(>0)$ and $L_{F_{1}}(>0)$ such that

$$
\left|F_{i}\left(x_{1}(t)\right)-F_{i}\left(x_{2}(t)\right)\right| \leq L_{F_{i}}\left|x_{1}-x_{2}\right|, i=1,2, \forall x_{1}, x_{2} \in \mathbb{R} .
$$

$\left(\mathbf{H}_{2}\right)$ The kernels $K_{1}(t, \xi)$ and $K_{1}(t, \xi)$ are continuous on $I \times I$, and there exist two positive constants $K_{1}^{*}$ and $K_{2}^{*}$ in $I \times I$

such that

$$
K_{i}^{*}=\sup _{t \in I} \int_{0}^{t}\left|K_{i}(t, \xi)\right| d \xi<\infty, i=1,2 .
$$


$\left(\mathbf{H}_{3}\right) g: I \rightarrow \mathbb{R}$ is continuous on $I$.

$\left(\mathbf{H}_{4}\right) h: C(I, \mathbb{R}) \rightarrow \mathbb{R}$ is continuous on $C(I)$ and there exist constant $0<L_{h}<$ 1 such that

$$
\left|h\left(x_{1}(t)\right)-h\left(x_{2}(t)\right)\right| \leq L_{h}\left|x_{1}-x_{2}\right|, \forall x_{1}, x_{2} \in C(I, \mathbb{R}), t \in I
$$

The following lemma yields the equivalence between the problem (1) and the integral equation. The proof for this lemma is neglected because it is similar to some classical proofs in the literature.

Lemma 3. The function $x \in C(I, \mathbb{R})$ be a solution of the Caputo fractional VFIDE (1) if and only if $x$ is a solution of the integral equation

$$
\begin{aligned}
x(t)= & x_{0}+h(x)+\frac{1}{\Gamma(\rho)} \int_{0}^{t}(t-s)^{\rho-1} g(s) d s \\
& +\frac{1}{\Gamma(\rho)} \int_{0}^{t}(t-s)^{\rho-1}\left\{\int_{0}^{s} K_{1}(s, \tau) F_{1}(x(\tau)) d \tau\right. \\
& \left.+\int_{0}^{1} K_{2}(s, \tau) F_{2}(x(\tau)) d \tau\right\} d s .
\end{aligned}
$$

Our first result is concerned with existence based on Theorem 4 .

Theorem 5. Suppose $\left(H_{1}\right)-\left(H_{4}\right)$ hold. If

$$
\Delta_{1}:=\left(L_{h}+\frac{\sum_{i=1}^{2} L_{F_{i}} K_{i}^{*}}{\Gamma(\rho+1)}\right)<1,
$$

then the Caputo fractional VFIDE (1) has at least one solution on I.

Proof. Let $C(I, \mathbb{R})$ be the space of the continuous functions $x$ on $I$ with the usual norm defined by

$$
\|x\|_{\infty}=\sup _{t \in I}|x(t)|
$$

Consider the ball

$$
B_{\gamma}=\left\{x \in C(I, \mathbb{R}):\|x\|_{\infty} \leq \gamma\right\} \subset C(I, \mathbb{R})
$$

Clearly, $B_{\gamma}$ is nonempty convex closed subset of $C(I, \mathbb{R})$. Choose $\gamma$ such that $\gamma \geq \frac{\Delta_{2}}{1-\Delta_{1}}$, where $\Delta_{1}<1$, 


$$
\Delta_{2}:=\mu_{0}+\frac{\mu_{g}+\sum_{i=1}^{2} \mu_{F_{i}} K_{i}^{*}}{\Gamma(\rho+1)}
$$

$\mu_{g}:=\sup _{t \in[0,1]}|g(t)|, \mu_{0}:=\left|x_{0}\right|+\mu_{h}, \mu_{h}=|h(0)|, \mu_{F_{1}}:=\left|F_{1}(0)\right|$ and $\mu_{F_{2}}:=$ $\left|F_{2}(0)\right|$.

In view of Lemma 3, the equivalent fractional integral equation to Caputo fractional VFIDE (1) can be written as operator equation as follows

$$
x=P x+Q x, \quad x \in B_{\gamma} \subset C(I, \mathbb{R}),
$$

where $P$ and $Q$ are two operators defined on $B_{\gamma}$ by

$$
\begin{aligned}
(P x)(t)= & \frac{1}{\Gamma(\rho)} \int_{0}^{t}(t-s)^{\rho-1}\left(\int_{0}^{s} K_{1}(s, \tau) F_{1}(x(\tau)) d \tau\right. \\
& \left.+\int_{0}^{1} K_{2}(s, \tau) F_{2}(x(\tau)) d \tau\right) d s
\end{aligned}
$$

and

$$
(Q x)(t)=x_{0}+h(x)+\frac{1}{\Gamma(\rho)} \int_{0}^{t}(t-s)^{\rho-1} g(s) d s .
$$

Now, we obtain the fixed point of the operator equation (8) by using the conditions of Theorem 6 as in the following steps:

Step 1: In this step, we show that, $P x+Q v \in B_{\gamma}$ for each $x, v \in B_{\gamma}$.

By $\left(\mathrm{H}_{1}\right)$ and for any $x, v \in B_{\gamma}$, we have

$$
\begin{aligned}
\left|F_{i}(x(t))\right| & \leq\left|F_{i}(x(t))-F_{i}(0)\right|+\left|F_{i}(0)\right| \\
& \leq L_{F_{i}}\|x\|_{\infty}+\left|F_{i}(0)\right| \\
& \leq L_{F_{i}} \gamma+\mu_{F_{i}}, \quad \text { for all } i=1,2
\end{aligned}
$$

and

$$
\begin{aligned}
|h(v(t))| & \leq|h(v(t))-h(0)|+|h(0)| \\
& \leq L_{h}\|v\|_{\infty}+|h(0)| \\
& \leq L_{h} \gamma+\mu_{h} .
\end{aligned}
$$

Let $x, v \in B_{\gamma}$. Then

$$
\begin{aligned}
& |(P x)(t)+(Q v)(t)| \\
\leq & \frac{1}{\Gamma(\rho)} \int_{0}^{t}(t-s)^{\rho-1}\left(\int_{0}^{s}\left|K_{1}(s, \tau)\right|\left|F_{1}(x(\tau))\right| d \tau\right.
\end{aligned}
$$




$$
\begin{aligned}
& \left.+\int_{0}^{1}\left|K_{2}(s, \tau)\right|\left|F_{2}(x(\tau))\right| d \tau\right) d s \\
& +\left|x_{0}\right|+|h(v)|+\frac{1}{\Gamma(\rho)} \int_{0}^{t}(t-s)^{\rho-1}|g(s)| d s \\
& \leq \quad \mu_{0}+L_{h} \gamma+\frac{\mu_{g}+\sum_{i=1}^{2}\left(L_{F_{i}} \gamma+\mu_{F_{i}}\right) K_{i}^{*}}{\Gamma(\rho+1)} t^{\rho},
\end{aligned}
$$

which implies

$$
\begin{aligned}
& \|P x+Q v\|_{\infty} \\
\leq & \mu_{0}+\frac{\mu_{g}+\sum_{i=1}^{2} \mu_{F_{i}} K_{i}^{*}}{\Gamma(\rho+1)}+\left(L_{h}+\frac{\sum_{i=1}^{2} L_{F_{i}} K_{i}^{*}}{\Gamma(\rho+1)}\right) \gamma \\
\leq & \Delta_{2}+\Delta_{1} \gamma \leq \gamma .
\end{aligned}
$$

Consequently,

$$
P x+Q v \in B_{\gamma}
$$

Step 2: In this step, we show that $Q$ is contraction on $B_{\gamma}$. Let $x, x^{*} \in B_{\gamma}$. It follows from $\left(\mathrm{H}_{4}\right)$ that

$$
\begin{aligned}
\left\|Q x-Q x^{*}\right\|_{\infty} & =\sup _{t \in I}|Q x(t)-Q x(t)|=\sup _{t \in I}\left|h(x(t))-h\left(x^{*}(t)\right)\right| \\
& \leq L_{h}\left\|x-x^{*}\right\|_{\infty} .
\end{aligned}
$$

Since $L_{h}<1, Q$ is contraction mapping.

Step 3: In this step, we show that, $P$ is completely continuous on $B_{\gamma}$.

First, we show that $P$ is continuous. Let $\left(x_{n}\right)$ be a sequence such that $x_{n} \rightarrow x$ in $C(I, \mathbb{R})$. Then for each $x_{n}, x \in C(I, \mathbb{R})$ and for any $t \in I$, we have

$$
\begin{aligned}
& \left|\left(P x_{n}\right)(t)-(P x)(t)\right| \\
\leq & \frac{1}{\Gamma(\rho)} \int_{0}^{t}(t-s)^{\rho-1}\left(\int_{0}^{s}\left|K_{1}(s, \tau)\right|\left|F_{1}\left(x_{n}(\tau)\right)-F_{1}(x(\tau))\right| d \tau\right. \\
& \left.+\int_{0}^{1}\left|K_{2}(s, \tau)\right|\left|F_{2}\left(x_{n}(\tau)\right)-F_{2}(x(\tau))\right| d \tau\right) d s \\
\leq & \frac{\sum_{i=1}^{2} L_{F_{i}} K_{i}^{*}}{\Gamma(\rho+1)}\left\|x_{n}-x\right\|_{\infty} .
\end{aligned}
$$

Since $x_{n} \rightarrow x$ as $n \rightarrow \infty,\left\|P x_{n}-P x\right\|_{\infty} \rightarrow 0$, as $n \rightarrow \infty$. This proves that $P$ is continuous on $C(I, \mathbb{R})$. 
Next, from Step 1, we observe that

$$
\begin{aligned}
& |(P x)(t)| \\
\leq & \frac{1}{\Gamma(\rho)} \int_{0}^{t}(t-s)^{\rho-1}\left(\int_{0}^{s}\left|K_{1}(s, \tau)\right|\left|F_{1}(x(\tau))\right| d \tau\right. \\
& \left.+\int_{0}^{1}\left|K_{2}(s, \tau)\right|\left|F_{2}(x(\tau))\right| d \tau\right) d s \\
\leq & \frac{\sum_{i=1}^{2}\left(L_{F_{i}} \gamma+\mu_{F_{i}}\right) K_{i}^{*}}{\Gamma(\rho+1)} t^{\rho} .
\end{aligned}
$$

Thus

$$
\|P x\|_{\infty} \leq \frac{\sum_{i=1}^{2}\left(L_{F_{i}} \gamma+\mu_{F_{i}}\right) K_{i}^{*}}{\Gamma(\rho+1)}
$$

This shows that $\left(P B_{\gamma}\right)$ is uniformly bounded.

Finally, we prove that $\left(P B_{\gamma}\right)$ is equicontinuous. Let $x \in B_{\gamma}$. Then for $t_{1}, t_{2} \in I$ with $t_{1} \leq t_{2}$, we have

$$
\begin{aligned}
& \left|(P x)\left(t_{2}\right)-(P x)\left(t_{1}\right)\right| \\
= & \mid \frac{1}{\Gamma(\rho)} \int_{0}^{t_{2}}\left(t_{2}-s\right)^{\rho-1}\left(\int_{0}^{s}\left|K_{1}(s, \tau)\right|\left|F_{1}(x(\tau))\right| d \tau\right. \\
& \left.+\int_{0}^{1}\left|K_{2}(s, \tau)\right|\left|F_{2}(x(\tau))\right| d \tau\right) d s \\
& -\frac{1}{\Gamma(\rho)} \int_{0}^{t_{1}}\left(t_{1}-s\right)^{\rho-1}\left(\int_{0}^{s}\left|K_{1}(s, \tau)\right|\left|F_{1}(x(\tau))\right| d \tau\right. \\
& \left.+\int_{0}^{1}\left|K_{2}(s, \tau)\right|\left|F_{2}(x(\tau))\right| d \tau\right) d s \mid \\
\leq & \frac{1}{\Gamma(\rho)}\left(\int_{t_{1}}^{t_{2}}\left(t_{2}-s\right)^{\rho-1} \int_{0}^{s}\left|K_{1}(s, \tau)\right|\left|F_{1}(x(\tau))\right| d \tau d s\right. \\
& \left.+\int_{0}^{t_{1}}\left|\left(t_{2}-s\right)^{\rho-1}-\left(t_{1}-s\right)^{\rho-1}\right| \int_{0}^{s}\left|K_{1}(s, \tau)\right|\left|F_{1}(x(\tau))\right| d \tau d s\right) \\
& +\frac{1}{\Gamma(\rho)}\left(\int_{t_{1}}^{t_{2}}\left(t_{2}-s\right)^{\rho-1} \int_{0}^{s}\left|K_{2}(s, \tau)\right|\left|F_{2}(x(\tau))\right| d \tau d s\right. \\
& \left.+\int_{0}^{t_{1}}\left|\left(t_{2}-s\right)^{\rho-1}-\left(t_{1}-s\right)^{\rho-1}\right| \int_{0}^{s}\left|K_{2}(s, \tau)\right|\left|F_{2}(x(\tau))\right| d \tau d s\right),
\end{aligned}
$$

which implies

$$
\left|(P x)\left(t_{2}\right)-(P x)\left(t_{1}\right)\right| \leq \frac{\left(L_{F_{1}} \gamma+\mu_{F_{1}}\right) K_{1}^{*}}{\Gamma(\rho)}\left(\int_{t_{1}}^{t_{2}}\left(t_{2}-s\right)^{\rho-1} d s\right.
$$




$$
\begin{aligned}
& \left.+\int_{0}^{t_{1}}\left|\left(t_{2}-s\right)^{\rho-1}-\left(t_{1}-s\right)^{\rho-1}\right| d s\right) \\
& +\frac{\left(L_{F_{2}} \gamma+\mu_{F_{2}}\right) K_{2}^{*}}{\Gamma(\rho)}\left(\int_{t_{1}}^{t_{2}}\left(t_{2}-s\right)^{\rho-1} d s\right. \\
& \left.+\int_{0}^{t_{1}}\left|\left(t_{2}-s\right)^{\rho-1}-\left(t_{1}-s\right)^{\rho-1}\right| d s\right) \\
\leq \quad & \left(\frac{\left(L_{F_{1}} \gamma+\mu_{F_{1}}\right) K_{1}^{*}}{\Gamma(\rho)}+\frac{\left(L_{F_{2}} \gamma+\mu_{F_{2}}\right) K_{2}^{*}}{\Gamma(\rho)}\right) \\
& \times\left(\frac{\left(t_{2}-t_{1}\right)^{\rho}}{\rho}+\frac{t_{1}^{\rho}}{\rho}-\frac{t_{2}^{\rho}}{\rho}+\frac{\left(t_{2}-t_{1}\right)^{\rho}}{\rho}\right) \\
\leq & \frac{2 \sum_{i=1}^{2}\left(L_{F_{i}} \gamma+\mu_{F_{i}}\right) K_{i}^{*}}{\Gamma(\rho+1)}\left(t_{2}-t_{1}\right)^{\rho}
\end{aligned}
$$

which tends to zero as $t_{2}-t_{1} \rightarrow 0$. So, $\left(P B_{\gamma}\right)$ is equicontinuous. Hence along with the Arzela-Ascoli theorem, it is concluded that $P: C(I, \mathbb{R}) \rightarrow C(I, \mathbb{R})$ is continuous and completely continuous.

An application of Theorem 4 shows that $P$ has a fixed point $x$ in $B_{\gamma}$ which is a solution of the Caputo fractional VFIDE (1).

The uniqueness result for the Caputo fractional VFIDE (1) will be proved by using Theorem 3 .

Theorem 6. Suppose $\left(H_{1}\right)-\left(H_{4}\right)$ hold. If

$$
\left(L_{h}+\frac{\sum_{i=1}^{2} L_{F_{i}} K_{i}^{*}}{\Gamma(\rho+1)}\right)<1,
$$

then the Caputo fractional VFIDE (1) has a unique solution on $I$.

Proof. Thanks to Lemma 3, the equivalent fractional integral equation to Caputo fractional VFIDE (1) can be written as operator equation as follows

$$
x=T x, \quad x \in C(I, \mathbb{R}),
$$

where the operator $T: C(I, \mathbb{R}) \rightarrow C(I, \mathbb{R})$ defined by

$$
\begin{aligned}
(T x)(t)= & x_{0}+h(x)+\frac{1}{\Gamma(\rho)} \int_{0}^{t}(t-s)^{\rho-1} g(s) d s \\
& +\frac{1}{\Gamma(\rho)} \int_{0}^{t}(t-s)^{\rho-1}\left(\int_{0}^{s} K_{1}(s, \tau) F_{1}(x(\tau)) d \tau\right.
\end{aligned}
$$




$$
\left.+\int_{0}^{1} K_{2}(s, \tau) F_{2}(x(\tau)) d \tau\right) d s
$$

for all $t \in I$. Let $x, x^{*} \in C(I, \mathbb{R})$. Then for for each $t \in I$ we have

$$
\begin{aligned}
& \left|T x(t)-T x^{*}(t)\right| \\
\leq \quad & \left|h(x(t))-h\left(x^{*}(t)\right)\right| \\
& +\frac{1}{\Gamma(\rho)} \int_{0}^{t}(t-s)^{\rho-1}\left(\int_{0}^{s} K_{1}(s, \tau)\left|F_{1}(x(\tau))-F_{1}\left(x^{*}(\tau)\right)\right| d \tau\right) d s \\
& +\frac{1}{\Gamma(\rho)} \int_{0}^{t}(t-s)^{\rho-1}\left(\int_{0}^{1} K_{2}(s, \tau)\left|F_{2}(x(\tau))-F_{2}\left(x^{*}(\tau)\right)\right| d \tau\right) d s \\
\leq \quad & L_{h}\left\|x-x^{*}\right\|_{\infty}+\frac{1}{\Gamma(\rho)} \int_{0}^{t}(t-s)^{\rho-1} K_{1}^{*} L_{F_{1}}\left\|x-x^{*}\right\|_{\infty} d s \\
& +\frac{1}{\Gamma(\rho)} \int_{0}^{t}(t-s)^{\rho-1} K_{2}^{*} L_{F_{2}}\left\|x-x^{*}\right\|_{\infty} d s \\
\leq & \left(L_{h}+\frac{K_{1}^{*} L_{F_{1}}+K_{2}^{*} L_{F_{2}}}{\Gamma(\rho+1)} t^{\rho}\right)\left\|x-x^{*}\right\|_{\infty},
\end{aligned}
$$

which implies

$$
\left\|T x-T x^{*}\right\|_{\infty} \leq\left(L_{h}+\frac{\sum_{i=1}^{2} L_{F_{i}} K_{i}^{*}}{\Gamma(\rho+1)}\right)\left\|x-x^{*}\right\|_{\infty} .
$$

The relation (9) shows that $T$ is contraction on $C(I, \mathbb{R})$. Hence, by the conclusion of Theorem 3, $T$ has a unique fixed point, which is solution of the Caputo fractional VFIDE (1).

\section{Approximate solution}

Here, we provide the approximate solution of the Caputo fractional VFIDE (1) which relies on the fractional Adomian decomposition technique.

First, we recall the classical Adomian decomposition technique where the solution of the proposed problem is obtained in the form of a series as

$$
x=\sum_{n=0}^{\infty} x_{n},
$$


and the nonlinear terms $F_{1}, F_{2}$ and $h$ are decomposed as

$$
F_{1}=\sum_{n=0}^{\infty} A_{n}, \quad F_{2}=\sum_{n=0}^{\infty} B_{n}, h=\sum_{n=0}^{\infty} D_{n}
$$

where $A_{n}, B_{n}, D_{n}$ are Adomian polynomials for all $n \in \mathbb{N}$, and write

$$
\begin{gathered}
x=x(\lambda)=\sum_{n=0}^{\infty} \lambda^{n} x_{n}=x_{0}+\lambda x_{1}+\lambda^{2} x_{2}+\cdots+\lambda^{k} x_{k}+\cdots \\
F_{1}=F_{1}(\lambda)=\sum_{n=0}^{\infty} \lambda^{n} A_{n}=A_{0}+\lambda A_{1}+\lambda^{2} A_{2}+\cdots+\lambda^{k} A_{k}+\cdots \\
F_{2}=F_{2}(\lambda)=\sum_{n=0}^{\infty} \lambda^{n} B_{n}=B_{0}+\lambda B_{1}+\lambda^{2} B_{2}+\cdots+\lambda^{k} B_{k}+\cdots \\
h=h(\lambda)=\sum_{n=0}^{\infty} \lambda^{n} D_{n}=D_{0}+\lambda D_{1}+\lambda^{2} D_{2}+\cdots+\lambda^{k} D_{k}+\cdots
\end{gathered}
$$

By utilizing the previous formulas (12) (13), (14) and (15), we deduce that

$$
\begin{aligned}
& A_{n}=\frac{1}{n !}\left[\frac{d^{n}}{d \lambda^{n}}\left(F_{1} \sum_{i=0}^{\infty} \lambda^{i} x_{i}\right)\right]_{\lambda=0}, \\
& B_{n}=\frac{1}{n !}\left[\frac{d^{n}}{d \lambda^{n}}\left(F_{2} \sum_{i=0}^{\infty} \lambda^{i} x_{i}\right)\right]_{\lambda=0}, \\
& D_{n}=\frac{1}{n !}\left[\frac{d^{n}}{d \lambda^{n}}\left(h \sum_{i=0}^{\infty} \lambda^{i} x_{i}\right)\right]_{\lambda=0},
\end{aligned}
$$

where $x_{0}, x_{1}, x_{2}, \ldots$ are repeatedly specified by

$$
\left\{\begin{array}{c}
x_{0}(t)=x_{0}+I_{0^{+}}^{\rho}(g(t)) \\
x_{k+1}(t)=D_{k}+I_{0^{+}}^{\rho}\left(\int_{0}^{t} K_{1}(t, \xi) A_{k} d \xi\right) \\
+I_{0^{+}}^{\rho}\left(\int_{0}^{1} K_{2}(t, \xi) B_{k} d \xi\right), \quad k \geq 1 .
\end{array}\right.
$$


Now, we apply the modified Adomian decomposition method, Therefore, the scheme (16) gives

$$
\left\{\begin{array}{c}
x_{0}(t)=x_{0}+R_{1}(t) \\
x_{1}(t)=R_{2}(t)+D_{0}+I_{0^{+}}^{\rho}\left(\int_{0}^{t} K_{1}(t, \xi) A_{0} d \xi\right) \\
\quad+I_{0^{+}}^{\rho}\left(\int_{0}^{1} K_{2}(t, \xi) B_{0} d \xi\right) \\
x_{k+1}(t)=D_{k}+I_{0^{+}}^{\rho}\left(\int_{0}^{t} K_{1}(t, \xi) A_{k} d \xi\right) \\
+I_{0^{+}}^{\rho}\left(\int_{0}^{1} K_{2}(t, \xi) B_{k} d \xi\right), \quad k \geq 1 .
\end{array}\right.
$$

Now, we will study the convergence theorem of the solution based on the MADM.

Theorem 7. Assume that $\left(H_{1}\right)-\left(H_{4}\right)$ and (5) are satisfied, if the solution $x(t)=\sum_{i=0}^{\infty} x_{i}(t)$ and $\|x\|_{\infty}<\infty$ is convergent, then it converges to the exact solution of the Caputo fractional VFIDE (1).

Proof. The proof is similar to some works found in the literature see [8], so we omit it.

Example 1. Consider an integro-differential equation with Caputo fractional derivative

$$
\left\{\begin{array}{c}
{ }^{C} D_{0^{+}}^{\frac{1}{2}} x(t)=\frac{2}{\sqrt{\pi}}\left(\frac{4 t^{\frac{3}{2}}}{\Gamma(6)}+t^{\frac{1}{2}}\right)+\frac{t^{3}}{\Gamma(7)}+\frac{t}{\Gamma(8)} \\
+\frac{1}{4} \int_{0}^{t}(1+t-s) x(s) d s+\frac{5}{18} \int_{0}^{1} e^{s-t} x^{2}(s) d s
\end{array}\right.
$$

with the nonlocal condition

where

$$
x(0)=\frac{1}{4} x\left(\frac{1}{3}\right)
$$

$$
\begin{aligned}
\rho & =\frac{1}{2}, x_{0}=0, h(x(t))=\frac{1}{4} x\left(\frac{1}{3}\right), \\
g(t) & =\frac{2}{\sqrt{\pi}}\left(\frac{4 t^{\frac{3}{2}}}{\Gamma(6)}+t^{\frac{1}{2}}\right)+\frac{t^{3}}{\Gamma(7)}+\frac{t}{\Gamma(8)}, \\
K_{1}(t, \xi) & =\frac{1}{4}(1+t-\xi), K_{2}(t, \xi)=\frac{5}{18} e^{\xi-t} .
\end{aligned}
$$

Clearly, $L_{F_{1}}=L_{F_{2}}=1, L_{h}=\frac{1}{4}$.

$$
\mu_{g} \quad: \quad=\sup _{t \in[0,1]}|g(t)|=\|g\|_{\infty}
$$




$$
\begin{gathered}
=\frac{2}{\sqrt{\pi}}\left(\frac{4}{\Gamma(6)}+1\right)+\frac{1}{\Gamma(7)}+\frac{1}{\Gamma(8)} \\
=\frac{1302+\sqrt{\pi}}{630 \sqrt{\pi}}, \\
K_{1}^{*}=\frac{1}{4} \sup _{t \in I} \int_{0}^{t}|1+t-\xi| d \xi=\frac{1}{8} . \\
K_{2}^{*}=\frac{5}{18} \sup _{t \in I} \int_{0}^{t}\left|e^{\xi-t}\right| d \xi=\frac{5}{18} \sup _{t \in I} e^{-t} \int_{0}^{t}\left|e^{\xi}\right| d \xi \\
=\frac{5}{18}\left(1-\frac{1}{e}\right) .
\end{gathered}
$$

Hence,

$$
\Delta_{1}:=\left(L_{h}+\frac{\sum_{i=1}^{2} L_{F_{i}} K_{i}^{*}}{\Gamma(\rho+1)}\right) \approx 0.6<1 .
$$

As consequence of Theorem 6, then the problem (18)-(19) has a unique solution on $[0,1]$.

Applying the operator $I_{0^{+}}^{\frac{1}{2}}$ to both sides of equation (18-a), we get

$$
\begin{aligned}
x(t)= & \frac{1}{4} x\left(\frac{1}{3}\right)+I_{0^{+}}^{\frac{1}{2}}\left(\frac{2}{\sqrt{\pi}}\left(\frac{4 t^{\frac{3}{2}}}{\Gamma(6)}+t^{\frac{1}{2}}\right)+\frac{t^{3}}{\Gamma(7)}+\frac{t}{\Gamma(8)}\right) \\
& +I_{0^{+}}^{\frac{1}{2}}\left(\frac{1}{4} \int_{0}^{t}(1+t-s) x(s) d s\right)+I_{0^{+}}^{\frac{1}{2}}\left(\frac{5}{18} \int_{0}^{1} e^{s-t} x^{2}(s) d s\right) .
\end{aligned}
$$

Suppose

$$
\begin{aligned}
R(t)= & I_{0^{+}}^{\frac{1}{2}}\left(\frac{2}{\sqrt{\pi}}\left(\frac{4 t^{\frac{3}{2}}}{\Gamma(6)}+t^{\frac{1}{2}}\right)+\frac{t^{3}}{\Gamma(7)}+\frac{t}{\Gamma(8)}\right) \\
= & \frac{2}{\sqrt{\pi}} \frac{4}{\Gamma(6)}\left(I_{0^{+}}^{\frac{1}{2}} s^{\frac{3}{2}}\right)(t)+\frac{2}{\sqrt{\pi}}\left(I_{0^{+}}^{\frac{1}{2}} s^{\frac{1}{2}}\right)(t) \\
& +\frac{1}{\Gamma(7)}\left(I_{0^{+}}^{\frac{1}{2}} s^{3}\right)(t)+\frac{1}{\Gamma(8)}\left(I_{0^{+}}^{\frac{1}{2}} s\right)(t) \\
= & \frac{8 \Gamma\left(\frac{5}{2}\right)}{\sqrt{\pi} \Gamma(6) \Gamma(3)} t^{2}+\frac{2 \Gamma\left(\frac{3}{2}\right)}{\sqrt{\pi}} t+\frac{\Gamma(4)}{\Gamma(7) \Gamma\left(\frac{9}{2}\right)} t^{\frac{7}{2}}+\frac{1}{\Gamma\left(\frac{5}{2}\right)} t^{\frac{3}{2}}
\end{aligned}
$$

Now, we apply the modified Adomian decomposition method,

$$
R(t)=R_{1}(t)+R_{2}(t)
$$


where

$$
R_{1}(t)=\frac{8 \Gamma\left(\frac{5}{2}\right)}{\sqrt{\pi} \Gamma(6) \Gamma(3)} t^{2}
$$

and

$$
R_{2}(t)=\frac{2 \Gamma\left(\frac{3}{2}\right)}{\sqrt{\pi}} t+\frac{\Gamma(4)}{\Gamma(7) \Gamma\left(\frac{9}{2}\right)} t^{\frac{7}{2}}+\frac{1}{\Gamma\left(\frac{5}{2}\right)} t^{\frac{3}{2}} .
$$

The modified recursive relation

$$
\begin{aligned}
& x_{0}(t)=R_{1}(t)=\frac{8 \Gamma\left(\frac{5}{2}\right)}{\sqrt{\pi} \Gamma(6) \Gamma(3)} t^{2}, \\
& x_{1}(t)=R_{2}(t)+I_{0^{+}}^{\frac{1}{2}}\left(\frac{1}{4} \int_{0}^{t}(1+t-s) A_{0}(s) d s\right) \\
& +I_{0^{+}}^{\frac{1}{2}}\left(\frac{5}{18} \int_{0}^{1} e^{s-t} B_{0}(s) d s\right)+D_{0}(t) \\
& =\frac{2 \Gamma\left(\frac{3}{2}\right)}{\sqrt{\pi}} t+\frac{\Gamma(4)}{\Gamma(7) \Gamma\left(\frac{9}{2}\right)} t^{\frac{7}{2}}+\frac{1}{\Gamma\left(\frac{5}{2}\right)} t^{\frac{3}{2}} \\
& +I_{0^{+}}^{\frac{1}{2}}\left(\frac{1}{4} \int_{0}^{t}(1+t-s) x_{0}(s) d s\right) \\
& +I_{0^{+}}^{\frac{1}{2}}\left(\frac{5}{18} \int_{0}^{1} e^{s-t} x_{0}(s) d s\right)+\frac{1}{4} x_{0}\left(\frac{1}{3}\right) \\
& =\frac{2 \Gamma\left(\frac{3}{2}\right)}{\sqrt{\pi}} t+\frac{\Gamma(4)}{\Gamma(7) \Gamma\left(\frac{9}{2}\right)} t^{\frac{7}{2}}+\frac{1}{\Gamma\left(\frac{5}{2}\right)} t^{\frac{3}{2}} \\
& +I_{0^{+}}^{\frac{1}{2}}\left(\frac{1}{4} \int_{0}^{t}(1+t-s) \frac{8 \Gamma\left(\frac{5}{2}\right)}{\sqrt{\pi} \Gamma(6) \Gamma(3)} s^{2} d s\right) \\
& +I_{0^{+}}^{\frac{1}{2}}\left(\frac{5}{18} \int_{0}^{1} e^{s-t} \frac{8 \Gamma\left(\frac{5}{2}\right)}{\sqrt{\pi} \Gamma(6) \Gamma(3)} s^{2} d s\right) \\
& +\frac{1}{4} \frac{8 \Gamma\left(\frac{5}{2}\right)}{\sqrt{\pi} \Gamma(6) \Gamma(3)}\left(\frac{1}{3}\right)^{2} \\
& =0 \text {, } \\
& x_{2}(t)=0, \\
& x_{n}(t)=0 .
\end{aligned}
$$


Therefore, the obtained solution is

$$
x(t)=\sum_{i=0}^{\infty} x_{i}(t)=\frac{8 \Gamma\left(\frac{5}{2}\right)}{\sqrt{\pi} \Gamma(6) \Gamma(3)} t^{2} .
$$

\section{References}

[1] M.S. Abdo, S.K. Panchal, Some new uniqueness results of solutions to nonlinear fractional integro-differential equations, Annals of Pure and Applied Mathematics, 16, No 1 (2018), 345-352.

[2] M.S. Abdo, A.M. Saeed, H.A. Wahash, S.K. Panchal, On nonlocal problems for fractional integro-differential equation in Banach space, European Journal of Scientific Research, 151, No 3 (2019), 320-334.

[3] M.S. Abdo, S.K. Panchal. Fractional integro-differential equations involving $\psi$-Hilfer fractional derivative, Advances in Applied Mathematics and Mechanics, 11, No 2 (2018), 338-359.

[4] M.S. Abdo, A.M. Saeed, S.K. Panchal, Caputo fractional integro-differential equation with nonlocal conditions in Banach space,International Journal of Applied Mathematics, 32, No 2 (2019), 279-288; doi:10.12732/ijam.v32i2.9.

[5] H.A. Wahash, M.S. Abdo, S.K. Panchal, Fractional integrodifferential equations with nonlocal conditions and generalized Hilfer fractional derivative, Ufa Mathematical Journal, 11, No 1 (2019), 3-21.

[6] M.S. Abdo, H.A. Wahash, S.K. Panchal, Ulam-Hyers-Mittag-Leffler stability for a $\psi$-Hilfer problem with fractional order and infinite delay, Results in Applied Mathematics, No 7 (2020), ID 100115.

[7] G. Adomian, A review of the decomposition method in applied mathematics, J. Math. Anal. Appl., 135, No 2 (1988), 501-544.

[8] A.H. Ahmed, M.S. Abdo, K.B. Ghadle, Existence and uniqueness results for Caputo fractional integro-differential equations, J. Korean Soc. for Industrial and Applied Mathematics, 22, No 3 (2018), 163-177.

[9] R.P Agarwal, M. Belmekki, M. Benchohra, A survey on semilinear differential equations and inclusions involving Riemann Liouville 
fractional derivative, Adv. Difference Equ., 47 (2009), Art. 981728; doi:10.1155/2009/981728.

[10] A. Anguraj, P. Karthikeyan, M. Rivero, J.J. Trujillo, On new existence results for fractional integro-differential equations with impulsive and integral conditions, Compu. Math. Appl., 66, No 12 (2014), 2587-2594.

[11] R.P. Agarwal, S.K. Ntouyas, B. Ahmad and M.S. Alhothuali, Existence of solutions for integro-differential equations of fractional order with non-local three-point fractional boundary conditions, Adv. Differ. Eq., 128 (2013).

[12] G. Adomian, R. Rach, Inversion of nonlinear stochastic operators, J. Math. Anal. Appl., 91, No 1 (1983).

[13] G. Adomian, D. Sarafyan, Numerical solution of differential equations in the deterministie limit of stochastic theory, Appl. Math. Comput., 8 (1981), 111-119.

[14] B. Ahmad, S. Sivasundaram, Some existence results for fractional integrodifferential equations with nonlinear conditions, Commun. Appl. Anal., 12, No 2 (2008), 107-112.

[15] D.V. Bayram, A. Dascpmoglu, A method for fractional Volterra integrodifferential equations by Laguerre polynomials, Adv. Differ. Equ., 2018, No 1 (2018), ID 466.

[16] H. Bazgir, B. Ghazanfari , Existence of solutions for fractional integrodifferential equations with non-local boundary conditions, Math. Comput. Appl., 23, No 3 (2018), ID 36.

[17] J.S. Duan, R. Rach, D. Baleanu, A.M. Wazwaz, A review of the Adomian decomposition method and its applications to fractional differential equations, Commun. Frac. Calc., 3, No 2 (2012), 73-99.

[18] P. Das, S. Rana, H. Ramos, Homotopy perturbation method for solving Caputo-type fractional order Volterra-Fredholm integro-differential equations, Comput. Math. Meth., 1, No 5 (2019), e1047.

[19] P. Das, S. Rana, H. Ramos, A perturbation-based approach for solving fractional-order Volterra-Fredholm integro differential equations and its convergence analysis, Int. J. Comput. Math. (2019), 1-21. 
[20] A.A. Elbeleze, A. Kilicman, B.M. Taib, Approximate solution of integrodifferential equation of fractional (arbitrary) order, J. King Saud Univ. Sci., 28, No 1 (2016), 61-68.

[21] J.H. He, Approximate analytical solution for seepage flow with fractional derivatives in porous media, Comput. Methods Appl. Mech. Eng., 167, No 1-2 (1998), 57-58.

[22] J.H. He, Some applications of non linear fractional differential equations and their approximations, Bull. Sci. Technol., 15, No 2 (1999), 86-90.

[23] H.N.A. Ismail, I.K. Youssef, T.M. Rageh, Modification on Adomian decomposition method for solving fractional Riccati differential equation, Intern. Advanced Research J. in Sci. Engin. and Technology, 4, No 12 (2017).

[24] A. Kilbas, H. Srivastava, J. Trujillo, Theory and Applications of Fractional Differential Equations, North-Holland Math. Stud., Elsevier, Amsterdam, 2006.

[25] Z. Laadjal, Q.H. Ma, Existence and uniqueness of solutions for nonlinear Volterra-Fredholm integro-differential equation of fractional order with boundary conditions, Math. Meth. Appl. Sci., (2019), 1-13; DOI:10.1002/mma.5845.

[26] R. Mittal, R. Nigam, Solution of fractional integro-differential equations by Adomian decomposition method, Int. J. Appl. Math. Mech., 4, No 2 (2008), 87-94.

[27] R. Rach, On the Adomian decomposition method and comparisons with Picard's method, J. Math. Anal. Appl., 128, No 2 (1987), 480-483.

[28] D.R. Smart, Fixed Point Theorems, Cambridge Univ. Press 66 (1980).

[29] S.S. Redhwan, S.L. Shaikh, M.S. Abdo, Implicit fractional differential equation with anti-periodic boundary condition involving CaputoKatugampola type, AIMS Mathematics, 5, No 4 (2020), 3714-3730.

[30] J.V.D.C. Sousa, D.D.S. Oliveira, C.E. de Oliveira, On the existence and stability for noninstantaneous impulsive fractional integrodifferential equation, Math. Meth. Appl. Sci., 42, No 4 (2019), 1249-1261.

[31] H.A. Wahash, M.S. Abdo and S.K. Panchal, An existence result for fractional integro-differential equations in Banach space, J. of Mathematical Extension, 13, No 3 (2019), 19-33. 
[32] J. Wu, Y. Liu, Existence and uniqueness of solutions for the fractional integro-differential equations in Banach spaces, Electron. J. Diff. Equ., 129 (2009), 1-8.

[33] A.M. Wazwaz, A reliable modification of Adomian decomposition method, Appl. Math. Comput., 102, No 1 (1999), 77-86.

[34] E.A.A. Ziada, Solution of coupled system of Cauchy problem of nonlocal differential equations, Electronic J. Math. Anal. Appl., 8, No 2 (2020), 220-230. 